\title{
La démocratisation de I'enseignement en France depuis la Seconde Guerre mondiale
}

\section{Antoine Prost}

Cet article dresse un bilan de la démocratisation de l'enseignement en France. Il rappelle les origines de cette politique dans la première moitié du siècle. Il explique pourquoi elle a attendu les années 1959-63 pour se réaliser: c'est alors la réforme de l'école moyenne et la première vague de croissance, jusqu'au milieu des années '70. Il analyse ensuite la seconde vague de croissance qui bouleverse la morphologie des lycées à partir de 1985. La question des résultats se pose alors: ces politiques ont-elles réduit les inégalités sociales devant l'école?

La réponse est nuancée. L'ouverture du collège, puis des lycées, a facilité l'accès des enfants modestes aux études et réduit globalement les inégalités. Mais, à l'intérieur de ce mouvement d'ensemble, l'inégalité entre les différentes filières a évolué de façon variable: elle s'est probablement réduite jusqu'au milieu des années '60; elle s'est ensuite stabilisée, voire creusée, en raison des procédures sélectives et de l'orientation instituée en 1973; enfin, les décisions de 1985 ont sans doute un peu relancé la démocratisation. Mais les espoirs dont elle était porteuse n'ont pas été satisfaits.

S'il fallait résumer d'un mot l'évolution de l'enseignement au $20^{\mathrm{e}}$ siècle, celui de "démocratisation» s'imposerait. La fortune de ce terme apparu au lendemain de la Grande Guerre ${ }^{1}$ tient à son ambiguïté même: il désigne en effet simultanément deux évolutions en partie liées, en partie différentes.

En un premier sens, la démocratisation est l'extension progressive à toute la population de scolarités initialement réservées à une minorité; la démocratisation de l'enseignement s'apparente alors à celle des vacances ou de l'automobile et elle se confond avec la croissance des effectifs scolaires et l'allongement des scolarités; l'augmentation des taux de scolarisation aux divers âges en fournit un bon indicateur. J'avais proposé d'appeler démocratisation «quantitative» cette évolution (Prost, 1986). Elle répond aussi bien au projet modernisateur qui cherche à élever le niveau de formation de la main-d'œuvre qu'au projet humaniste de ceux pour qui l'enseignement s'arrête toujours trop tôt. 
En un second sens, la démocratisation vise à réduire les inégalités sociales en donnant à tous les enfants les mêmes chances devant l'école, quel que soit le milieu auquel ils appartiennent. C'est un projet politique, inspiré par un souci de justice et d'égalité, qui vise à transformer la société elle-même. En réformant ce qui, dans l'école, organise la reproduction des inégalités sociales, on veut mettre fin à leur caractère hérité et les réduire à des différences de mérite, seules légitimes. J'avais proposé d'appeler «qualitative» cette démocratisation qui ne concerne plus seulement la généralisation de l'école, mais l'articulation entre ses diverses filières et la façon dont les divers groupes sociaux y accèdent.

Ces deux formes de démocratisation ont longtemps constitué une seule et même cause, avant qu'on ne prenne progressivement conscience du fait que l'une n'entraînait pas nécessairement l'autre. D'où une histoire complexe, que l'on se propose de retracer pour la France dans la seconde moitié du $20^{\text {e }}$ siècle, en confrontant les intentions des acteurs, les politiques mises en œuvre et leurs résultats.

\section{La Lente émergence du projet de démocratisation}

\section{Les origines}

L'idée de démocratisation naît de la prise de conscience des inégalités sociales devant l'école. Entre 1905 et 1910, apparaît ainsi, dans les congrès de la Ligue de l'enseignement et du Parti radical-socialiste, une critique sociale de la séparation du secondaire et du primaire. Ces deux ordres d'enseignement sont moins complémentaires que concurrents, puisque le secondaire inclut des classes élémentaires où il recrute le gros de ses élèves, et que le primaire développe, au-delà du Certificat d'études, des enseignements primaires supérieurs. Pour Buisson, qui présente à la Chambre en 1910 un projet de loi tendant à assurer l'égalité des enfants devant le droit à l'instruction, cette distinction pérennise la division de la société entre deux classes sociales: "ceux qui possèdent sans travailler et ceux qui travaillent sans posséder»; les bourses ne sont que "des exceptions consolantes» (cité par Gavoille, 1983, p. 350). D'où l'idée de substituer, aux deux ordres d'enseignement parallèles, une structure en cycles successifs, avec un enseignement élémentaire rigoureusement commun à tous les élèves. C'est l'idée de l'école unique, popularisée après la Guerre par un groupe d'anciens combattants appartenant à tous les ordres d'enseignement, les Compagnons de l'université nouvelle, développée aussi par un professeur socialiste, Zoretti, et reprise par le Cartel des gauches dans son programme en 1924.

Tel qu'il prend forme alors, le projet d'école unique comporte la suppression des classes élémentaires des établissements secondaires et la prolongation jusqu’à 14 ans de l'école élémentaire. Les meilleurs seuls poursuivraient au lycée, dont les cancres, même de bonne famille, seraient exclus. Ce projet conjugue une volonté de modernisation par la prolongation de la scolarité et la formation profession- 
nelle, une volonté démocratique de brassage social par l'école unique, et une volonté de justice sociale par une sélection fondée sur le mérite. Mais la volonté du gouvernement est trop faible en 1924 pour faire aboutir ce projet encore trop vague. Il est précisé par ses partisans en 1927, adopté par la Fédération générale de l'enseignement CGT puis par la CGT (Confédération générale du travail) elle-même en 1931 (Talbott, 1969).

Cependant, la concurrence entre le primaire supérieur et le secondaire se durcit. C'est en effet l'époque où les enfants nés pendant la Guerre atteignent 11-15 ans. Or ils sont deux fois moins nombreux que ceux des générations précédentes. Le problème se pose donc de remplir les classes, et singulièrement les classes de 6ème des lycées. ${ }^{2}$ Certains lycées acceptent alors des élèves avec des notes très inférieures à la moyenne, 6 ou 7 sur 20, pour éviter de fermer des classes. Les professeurs des lycées s'indignent de voir de bons élèves poursuivre leur scolarité dans des écoles primaires supérieures (EPS) au lieu de venir dans leurs propres classes: c'est pour eux une concurrence déloyale. Ils ne voient pas que le succès de leur rival repose sur une meilleure adaptation aux besoins des familles: moins long, le primaire supérieur offre une scolarité moins onéreuse en dépit de la gratuité du secondaire, réalisée précisément en 1930; plus concret, plus «scolaire», assuré par des maîtres plus proches des milieux populaires, il semble aussi plus accessible; enfin, ses diplômes ont, sur le marché du travail, une valeur plus certaine que le baccalauréat. C'est pourquoi le retour, à partir de 1930, à une situation démographique plus favorable, s'il permet au secondaire d'enregistrer une forte croissance, ne supprime pas pour autant la concurrence du primaire supérieur, comme l'atteste la sélectivité des examens qui lui donnent accès. Dans deux EPS de Marseille, on compte en 1935 respectivement 752 et 500 candidats pour 162 et 100 places disponibles (Briand \& Chapoulie, 1981).

Le Front populaire reprend le projet d'école unique. Il commence par prolonger de 13 à 14 ans l'obligation scolaire (loi du 9 août 1936) et son ministre de l'Education nationale, J. Zay, dépose en 1937 un projet d'ensemble. Mais il s'enlise à la Chambre. L'action de J. Zay se limite donc à ce qui ne dépend pas d'une loi: il unifie les programmes du premier cycle de l'enseignement du second degré et de l'enseignement primaire supérieur (de 11-12 à 15-16 ans) et il lance à titre expérimental, au niveau de la Gème, des classes d'observation dont l'objectif est de permettre l'orientation des élèves entre les diverses filières d'enseignement: lycées, primaire supérieur et enseignement professionnel. Puis la Guerre survient et Vichy suspend ces expériences. A la Libération, la Commission Langevin-Wallon (1944-47) propose une version plus élaborée du même projet, sans que les circonstances lui permettent d'aboutir.

\section{L'enlisement dans la concurrence, 1941-1959}

On peut alors se demander si la démocratisation sera un jour autre chose qu'un mot d'ordre rituellement ressassé. De 1944 à 1959, pas moins de quatorze projets de réforme en ce sens furent élaborés et avortèrent. L'instabilité de la IVe Ré- 
publique ne suffit pas à expliquer ce blocage qui résulte de l'affrontement de deux forces égales et également convaincues: le premier et le second degré. Partageant la même volonté de démocratisation, instituteurs et professeurs s'en font, en effet, deux idées totalement différentes entre lesquelles le choix s'avère impossible.

Pour les professeurs, la démocratisation signifie l'accès de tous à ce que la culture scolaire a de meilleur: les humanités classiques. Toute autre formule constituerait une démocratisation au rabais qu'ils refusent de toute leur énergie. Pour les instituteurs, cette conception est totalement inadaptée: un enseignement conçu et organisé pour une élite restreinte ne saurait devenir celui de toute la nation. Il faut concevoir une autre culture, plus moderne, plus technique, qui réponde aux attentes de la masse des élèves et qui ait sens pour leurs familles. Seuls les instituteurs, issus du peuple, proches du peuple et voués à former ses enfants, peuvent réussir dans cette tâche. A quoi les professeurs répondent qu'il n'y a qu'une seule culture, la leur, et que les instituteurs le savent bien puisqu'ils leur confient leurs propres enfants ...

Ce débat était un vrai débat. Il portait sur la durée et les contenus du cycle d'observation dont chacun s'accordait à faire l'élément central de la réforme. Pour les professeurs, retarder le début de l'apprentissage du latin était prendre parti contre les humanités et la culture: ils s'opposaient donc à un cycle d'observation trop long, voulant qu'il ne concerne que la $6^{\text {ème }}$, et non les deux classes de $6^{\text {ime }}$ et $5^{\text {ime }}$, et que tous les élèves abordent le latin en $6^{\text {ime }}$. Pour des raisons inverses, les instituteurs voulaient au contraire un cycle d'observation de deux années, et sans latin. Ce choix donnant le rôle central dans le cycle soit aux enseignants du second degré soit à ceux du premier, il impliquait des styles et des coutumes pédagogiques très différents. Surtout, il présentait des enjeux corporatifs majeurs sur lesquels s'affrontaient les syndicats d'enseignants des deux degrés, soucieux chacun d'élargir sa clientèle et de protéger ses débouchés.

Le conflit n'était pas seulement théorique; il se jouait aussi sur le terrain. En 1941, le ministre Carcopino, pour défendre le secondaire contre la concurrence déloyale des EPS, avait absorbé celles-ci dans le second degré, les transformant en collèges modernes. Que les professeurs de ces sections modernes ignorassent le latin était sans importance, puisqu'il n'était pas enseigné dans ces sections (étiquetées " $\mathrm{M}$ ) où l'on pratiquait deux langues vivantes. Mais les professeurs d'EPS n'étaient guère formés pour enseigner les langues. Les successeurs de Carcopino créèrent donc en 1952 à la première partie du baccalauréat une section moderne supplémentaire " $\mathrm{M}$ '» où l'une des deux langues vivantes était remplacée par des sciences naturelles que ces professeurs maîtrisaient mieux.

Cependant, Carcopino avait laissé subsister, dans certaines écoles primaires, des cours complémentaires (CC) qui dispensaient un enseignement primaire supérieur sur les mêmes programmes que les anciennes EPS, quoique avec un personnel moins qualifié. Or les $\mathrm{CC}$ sont très faciles à créer: la décision est décentralisée et ne demande guère de moyens; il suffit de libérer une ou deux salles de 
classe et deux instituteurs en chargeant un peu les classes élémentaires, ou en bénéficiant de quelques postes supplémentaires et de la bienveillance d'une municipalité pour les locaux. Le développement des CC représente une promotion à la fois pour les instituteurs qui y enseignent et pour les élèves qui trouvent ainsi, à proximité de chez eux et dans des conditions peu onéreuses, une possibilité de faire des études plus poussées, voire même d'aller jusqu'au baccalauréat s'ils sont admis en section M ou M' d'un lycée. En 1954, le proviseur du lycée de Chartres signale ainsi dans son rapport que 12 élèves admis en $6^{\text {ème }}$ ont préféré aller dans un cours complémentaire, mais il ne s'en plaint pas car, ajoute-t-il, «les cours complémentaires du département nous envoient, en seconde $\mathrm{M}^{\prime}$, de bons sujets, plus nombreux chaque année [...]». ${ }^{3}$ De fait, les deux sections $M$ et $M$ ' enregistrent une croissance rapide et elles fournissent en 1961 plus de la moitié des candidats reçus à la première partie du baccalauréat. ${ }^{4}$

On comprend le succès des cours complémentaires ainsi désenclavés. Stimulée par la concurrence des deux ordres d'enseignement, la scolarisation progresse rapidement et les effectifs des cours complémentaires rattrapent puis dépassent ceux des premiers cycles des lycées.

Tableau 1: Scolarisation dans le premier cycle, 1945-1962 (enseignement public)

\begin{tabular}{|l|c|c|c|}
\hline & $1945-46$ & $1959-60$ & $1961-62$ \\
\hline Premier cycle du secondaire & 206,6 & 458,5 & 528,1 \\
Cours complémentaires & 152,8 & 474,3 & 627,8 \\
Taux de scolarisation & $20,5 \%$ & $45,7 \%$ & $47,0 \%$ \\
\hline
\end{tabular}

Chiffres en milliers, source: Prost, 1981, p. 239.

Mais la concurrence entre degrés commence à engendrer des doubles emplois; une rationalisation s'impose. Dans un climat politique radicalement modifié par le passage de la $I V^{e}$ à la $V^{e}$ République, le gouvernement entreprend de prouver son efficacité dans le domaine de l'enseignement comme dans d'autres. L'heure des réformes est venue.

\section{De la réforme des collèges à celle des lycées}

\section{La mise en place du collège unique}

Pour moderniser le pays et lui rendre son rang, de Gaulle veut une économie forte et créative, qui suppose un main-d'œuvre globalement mieux formée et des techniciens et chercheurs beaucoup plus nombreux. Le thème du retard français en ce domaine est largement partagé: en 1965, Poignant estime que le nombre de diplômés de l'enseignement supérieur scientifique et technique est inférieur de près de moitié en France (2,2\% de la classe d'âge) à ce qu'il est en Grande-Bretagne (4,22\%), aux Etats-Unis (3,9\%) ou en URSS (4\%). La France n'est pas as- 
sez riche pour se permettre d'écarter des talents: il faut ouvrir largement le recrutement. Mais il n'est pas question de laisser submerger l'enseignement supérieur par des étudiants incapables de le suivre. La politique gaullienne tient en deux mots: démocratisation du premier cycle et sélection.

La première décision, prise en janvier 1959, consiste à prolonger l'obligation scolaire jusqu'à 16 ans pour les enfants nés après le 1 er janvier 1953 . Elle est accompagnée de la création d'un cycle d'observation en $6^{\text {ème }}$ et $5^{\text {ème }}$ qui ne change pratiquement rien aux programmes - le début du latin est repoussé d'un trimestre - mais qui contribue à accélérer la croissance de la scolarisation. Par une action vigoureuse, l'administration implante en effet des classes d'observation au plus près des familles, dans des écoles primaires. Mais les élèves poursuivent leur scolarité dans le type d'établissement, secondaire, primaire ou technique, où ils ont suivi le cycle d'observation. La démocratisation quantitative qui s'engage n'est donc pas une démocratisation qualitative.

Or le gouvernement affirme sa volonté d'établir une meilleure égalité des chances devant l'enseignement. Il faut donc tenter de rendre l'orientation effective. Au même moment (1962) une grande enquête de l'Institut national d'études démographiques souligne l'ampleur des inégalités. A réussite scolaire jugée également excellente par leurs instituteurs, $89 \%$ des enfants de cadres supérieurs poursuivent leurs études au lycée contre $42 \%$ des enfants d'ouvriers. Les pourcentages pour une réussite seulement bonne sont respectivement de $79 \%$ et $27 \%$, et ils tombent à $64 \%$, près des deux tiers encore, contre $14 \%$ pour une réussite moyenne. Pour une réussite franchement médiocre, plus de la moitié des enfants de cadres supérieurs passent en $6^{\text {ème }}(52 \%)$, contre $2 \%$ des enfants d'ouvriers (Girard \& Bastide, 1963, pp. 96-97). Le succès de quelques élèves de milieu modeste ne suffit plus à démentir ce constat irréfutable: l'entrée au lycée dépend du milieu social et non du mérite scolaire. L'année suivante, Bourdieu et Passeron (1964) font sensation en montrant que l'université est l'image renversée de la nation: «Un fils de cadre supérieur a quatre-vingts fois plus de chances d'entrer à l'université qu'un fils de salarié agricole et quarante fois plus qu'un fils d'ouvrier» (p. 14).

C'est dans ce contexte que s'est réalisée progressivement en France l'école moyenne. L'évolution prit une dizaine d'années. La décision capitale fut, en 1963, de créer des collèges de quatre ans, ajoutant aux deux années du cycle d'observation ( $6^{\text {ìme }}$ et $\left.5^{\text {ìme }}\right)$ deux années de cycle d'orientation ( $4^{\text {ème }}$ et $\left.3^{\text {ème }}\right)$. Ces collèges étaient de deux types, correspondant aux deux modèles antérieurs du premier cycle des lycées et des cours complémentaires ${ }^{5}$, mais la carte scolaire fut entièrement remaniée, avec la construction de plus de 2'500 collèges, la séparation physique du premier et du second cycle des lycées et le recrutement massif de nouveaux professeurs. Le budget de l'Education nationale fit plus que doubler, passant d'un peu plus de $7 \%$ du budget total à plus de $16 \%$ en 1965 , et presque $20 \%$ en 1977 . Progressivement, les différences entre les deux types de collèges s'estompèrent et, en leur sein, les différences entre filières, classiques, 
modernes de type lycée, modernes de type primaire supérieur. ${ }^{6}$ En 1975, une loi put donc instituer le collège unique et supprimer les filières du cycle d'observation.

Cette première vague de croissance a généralisé la scolarisation jusqu'à 16 ans. Les taux de scolarisation à 14,15 et 16 ans, qui étaient respectivement de $68,4 \%$, $53 \%$ et $43 \%$ en 1958 s'établissent à $97,2 \%, 91,7 \%$ et $69,9 \%$ en 1975 . Mais, alors que le projet de 1963 voulait que tous les élèves aillent au collège jusqu’à son terme, un nombre important d'entre eux s'arrête à mi-chemin et entre en apprentissage après la fin du cycle d'observation, ou poursuit dans un lycée d'enseignement professionnel trois années d'études sanctionnées par un CAP, un certificat d'aptitude professionnelle qui distingue les ouvriers qualifiés.

L’organisation scolaire est donc profondément modifiée. Les graphiques 1 et 2 ci-dessous illustrent les transformations à la fois morphologiques et quantitatives provoquées par la réforme des collèges. Aux établissements concurrents hérités du passé, se substitue ce qui devient un véritable système scolaire. L'école élémentaire s'unifie, les lycées renonçant à leurs "petites» classes ${ }^{7}$ pour ouvrir des classes plus prestigieuses. Les collèges s'imposent comme un niveau intermédiaire où, après le tronc commun du cycle d'observation, commencent à diverger des filières, l'orientation étant censée s'effectuer sur des critères scolaires et non selon les milieux sociaux d'origine. Un rameau se détache vers la formation professionnelle à la fin de la $5^{\text {ème }}$, mais les divisions principales s'effectuent après le cycle d'orientation, à la fin de la $3^{\text {ème. }}$. La réforme du second cycle du second degré (classes de 2de, lère et terminale) effectuée en 1965 organise alors trois filières principales, conduisant au baccalauréat d'enseignement général, au baccalauréat d'enseignement technique et à un nouveau diplôme professionnel, le Brevet d'études professionnelles (BEP), de même niveau que le CAP mais plus polyvalent. Des procédures d'orientation se mettent en place en 1973 pour affecter les élèves sortant du collège à ces diverses filières. 

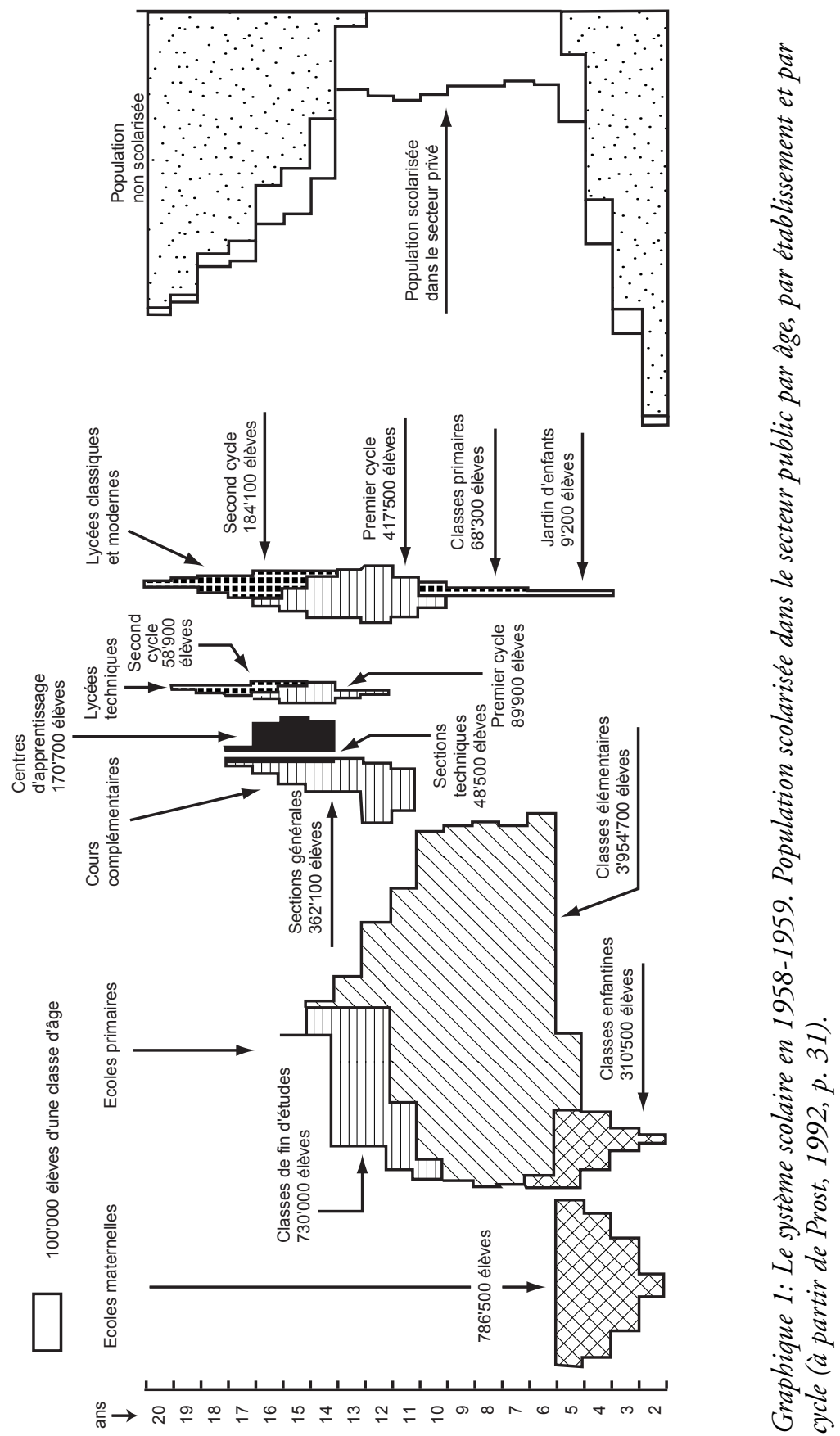


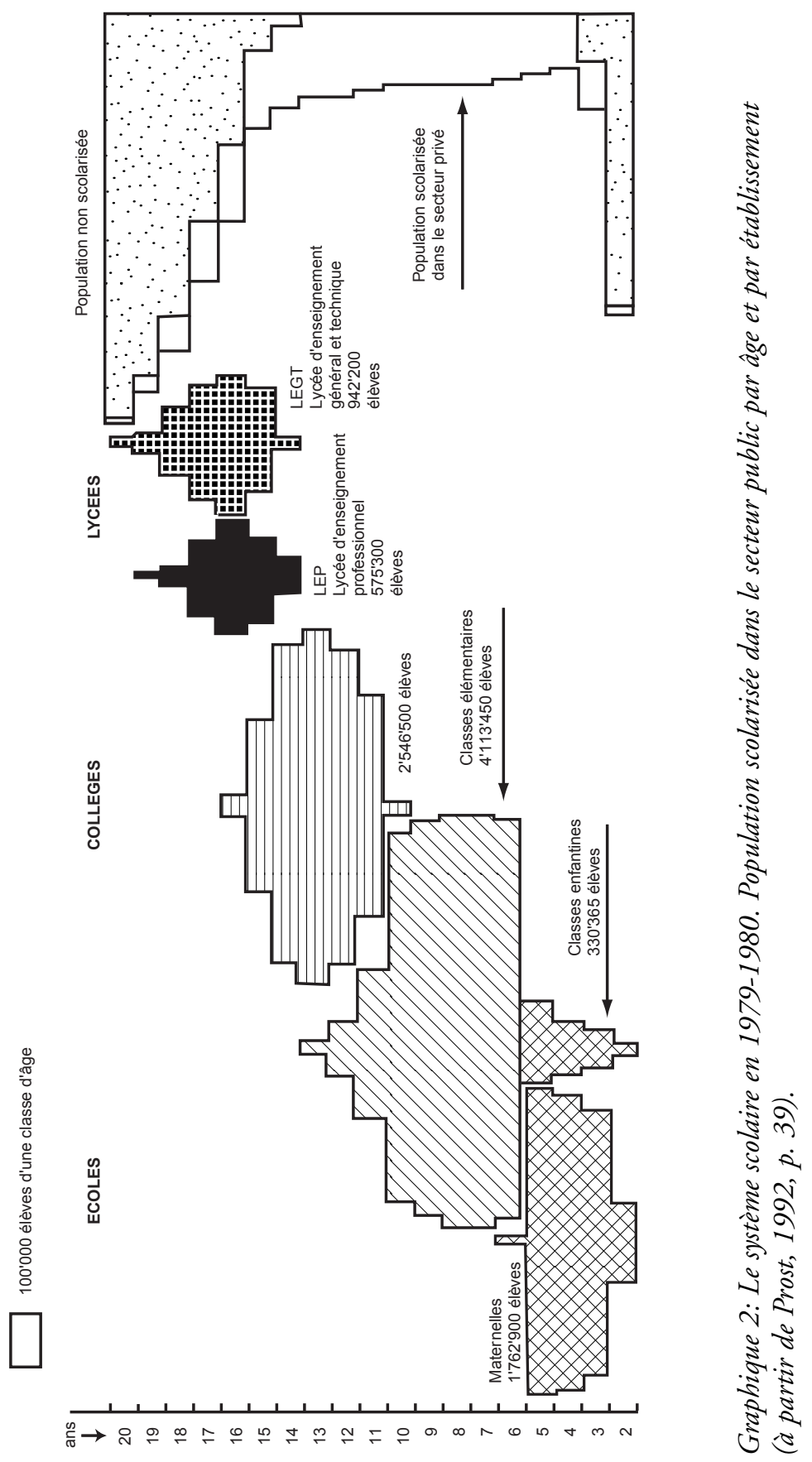




\section{La seconde vague de croissance du système éducatif}

Pendant une dizaine d'années, de 1975 à 1985, le système scolaire français ne connaît pas de réforme majeure. La scolarisation à 16 et 17 ans progresse lentement, et les lycées, désormais cantonnés aux trois classes de $2^{\text {de }}, 1^{\text {ere }}$ et terminale, enregistrent une croissance modérée, de l'ordre de 1 à $2 \%$ par an.

C'est alors qu'au plus profond de la crise économique, le gouvernement socialiste décide de relever massivement le niveau de formation pour affronter la concurrence internationale. Le Japon, où la scolarité de second cycle est généralisée, sert d'exemple. L'objectif affiché de cette nouvelle politique est de conduire $80 \%$ de la classe d'âge «au niveau du baccalauréat en l'an 2000». Les enseignements professionnels sont directement concernés, avec la création d'un nouveau baccalauréat professionnel destiné à former des ouvriers très qualifiés.

Cette réforme entraîne de profonds changements. A la rentrée de 1990, les lycées accueillent 363'000 élèves de plus qu'à celle de 1985. La croissance est de $30 \%$. Les taux de scolarisation progressent plus rapidement même qu'au cours du premier cycle de croissance: pour 16 ans, ils passent de $85,5 \%$ à 96,4\% entre 1982 et 1995 , de $71,9 \% \%$ à 92,3\% pour 17 ans et, pour 18 ans, de 45,1\% à $84,2 \%$ (étudiants inclus). Les lycées professionnels cessent de préparer leurs élèves aux CAP et basculent vers l'aval. La préparation aux BEP devient pour plus de la moitié des élèves l'antichambre du nouveau baccalauréat professionnel. De ce fait, le taux d'accès au baccalauréat qui était de 38,7\% pour les enfants nés en 1966, est de 62,8\% pour leurs successeurs nés 13 ans plus tard, en 1979. Le graphique 3 ci-dessous montre l'impact, sur la morphologie et la taille du système scolaire, de cette réforme et de la seconde vague de croissance qu'elle a provoquée dans les lycées.

La rapidité de ces changements est paradoxale. Ils s'accomplissent en effet entre 1985 et 1990 pour l'essentiel, dans une conjoncture beaucoup moins favorable que la prospérité des booming sixties qui avait facilité la croissance des collèges. La crise est installée et réduit les budgets, les crédits de l'Education nationale passant de 3,9\% du PIB en 1983 à 3,4\% en 1989; le recrutement de nouveaux professeurs est rendu difficile par la médiocre image du métier, son niveau de rémunération et surtout, dans les matières scientifiques, par la pénurie de candidats, voire de diplômés disponibles; les constructions tardent, car il faut du temps aux régions pour assumer cette responsabilité dont l'Etat s'est déchargé en 1985 par la décentralisation. La croissance entraîne donc la surcharge des classes: la proportion de classes du second cycle accueillant 35 élèves ou plus, qui était de 9,4\% à la rentrée de 1982, atteint 39,4\% à celle de 1988 . Une évolution moins brutale aurait suscité moins de tensions. Pourquoi cette seconde "explosion» scolaire, paradoxalement plus rapide que la première?

C'est que la première réforme, celle des collèges, avait profondément modifié le mode d'affectation des élèves dans les classes, transformant un système libéral en système réglementé. Auparavant, les choix d'orientation scolaire - mettre son enfant au lycée ou au CC - relevaient formellement des familles; ils s'effectuaient 


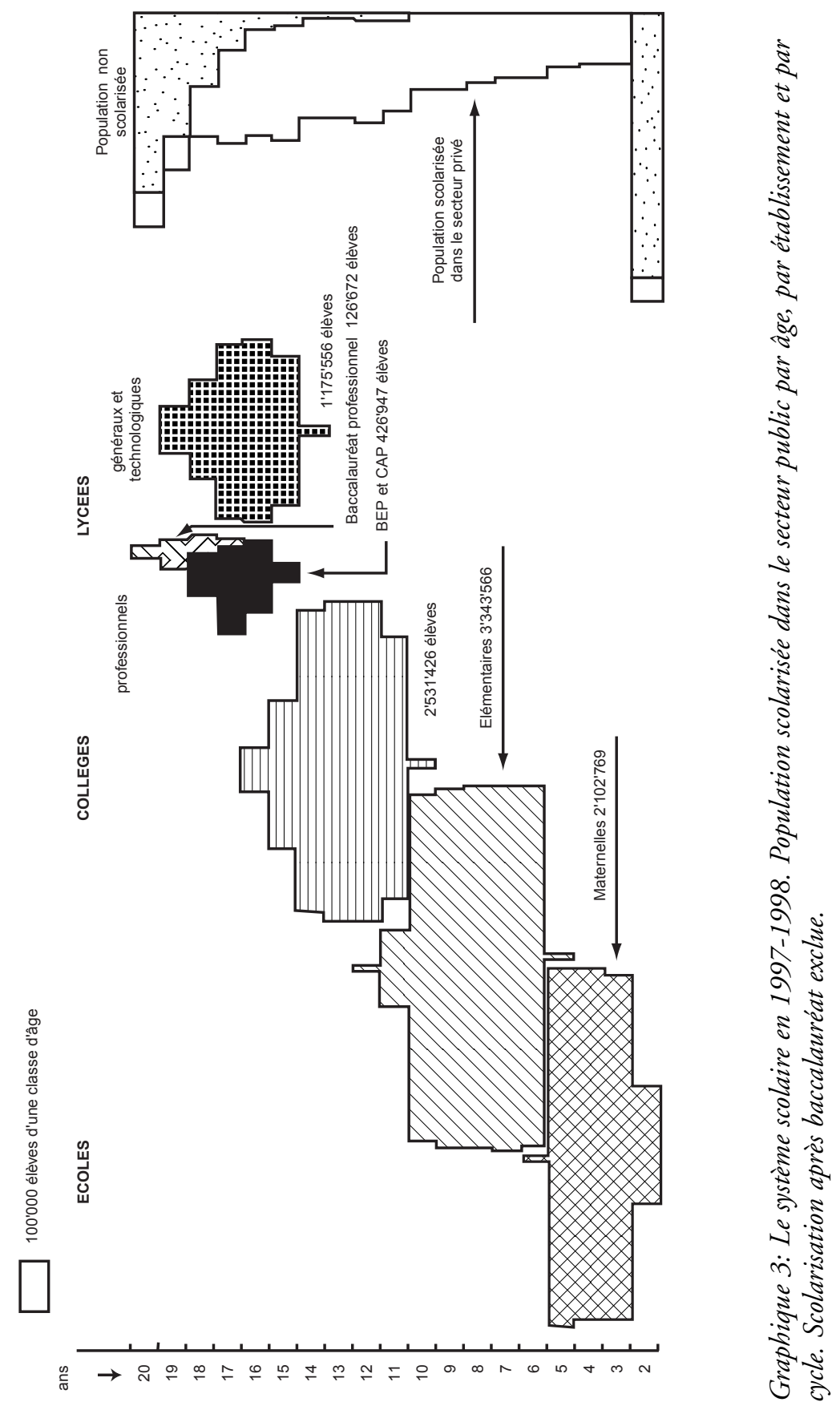


avant l'école et en dehors d'elle. La création du collège unique transfere cette responsabilité de la famille à l'école. L'élève est affecté à tel ou tel établissement en fonction de son domicile, c'est ce que l'on appelle la «sectorisation». L'administration décide, à la fin du collège, s'il sera admis en seconde, et dans quelle seconde, c'est l'orientation; elle engendre d'inévitables conflits avec les familles et oblige à imaginer de complexes procédures d'appel pour les gérer. Cette réglementation est particulièrement contraignante: en 1983-84, alors que 78,3\% des parents d'élèves de $3^{\text {ème }}$ demandent que leur enfant soit admis en seconde, $60,6 \%$ seulement voient leur vœu exaucé (Prost, 1998).

L'effet de ces contraintes sur le système éducatif a été généralement sous-estimé. Elles ont renforcé la sélection; la précocité fournissant un critère apparemment objectif pour une orientation positive, l'âge des élèves des lycées s'est abaissé. Paradoxalement, les redoublements ont augmenté, notamment en classe de $3^{\text {ème }}$, dans l'espoir d'éviter des orientations trop pénalisantes. Les familles ont contourné les refus que leur opposait l'enseignement public en recourant aux établissements privés: alors que ces établissements scolarisent le quart des élèves du second degré, plus du tiers y font un passage à un moment ou à un autre de leur scolarité (Langouet \& Leger, 1991). On s'explique ainsi la mobilisation massive de 1983-84 contre le projet Savary qui visait à intégrer l'enseignement privé dans le service public d'Education: l'immense majorité (88\%) des parents d'élèves des établissements publics eux-mêmes souhaitaient pouvoir choisir l'établissement où placer leur enfant et pouvoir changer en cours d'année en cas de difficulté (79\%), éventuellement pour un établissement privé (58\%). ${ }^{8}$ Alors que l'opinion aspirait à la déréglementation, le projet Savary semblait étendre et renforcer la réglementation. D'où son refus.

D'où, aussi, la vigueur de la croissance à partir de 1985. L'objectif de $80 \%$ de la classe d'âge "au niveau du baccalauréat en l'an 2000" s'accompagne de la fermeture des filières de dérivation qui accueillaient les élèves au milieu du collège et de l'abandon, au moins partiel, des procédures qui freinaient l'accès au lycée depuis 1973. La croissance des collèges avait répondu à la fois à une demande et à une offre d'enseignement, l'administration s'étant donné les moyens de sa politique. La croissance des lycées répond, elle aussi, à une volonté politique affichée, mais qui se traduit moins par une augmentation, lente à venir, des capacités d'enseignement que par une réorientation du dispositif et la libération d'une demande jusqu'alors contrainte par la sectorisation et l'orientation.

\section{L'enseignement français s'est-il démocratisé?}

\section{Questions de méthode et de définition}

Pour savoir si cette croissance et ces transformations ont répondu aux objectifs qui étaient ceux des promoteurs de l'école unique entre les deux guerres, puis des réformateurs des années ' 60 , il est nécessaire d'évaluer les inégalités sociales 
devant l'école et ses diverses filières. Or cette mesure présente plusieurs difficultés.

La première est l'identification des catégories sociales auxquelles appartiennent les élèves. En effet, les professions indiquées par l'administration sont approximatives et elles peuvent différer lourdement de celles constatées par des enquêteurs. Chez les intéressés qui les déclarent, peuvent jouer des désirs de valorisation ou d'esquive. L'administration des établissements scolaires code ces professions déclarées selon des nomenclatures variables qui rendent les chiffres peu fiables. Dans les statistiques nationales, les proportions d'enfants d'ouvriers dans les classes augmentent de plusieurs points entre 1963-64 et 1967-68, mais celles d'enfants d'employés diminuent d'autant: on ne peut écarter l'hypothèse que ces mouvements s'expliquent par des changements de codification entre les deux dates. L'évolution sociale que la statistique semble prouver pourrait donc être illusoire. ${ }^{9}$ Merllié (2000), en confrontant les résultats des codages effectués par les établissements scolaires et par des enquêteurs sur la même population, aboutit à des résultats qui alimentent le scepticisme; pour les employés, où l'imprécision est la plus grande, les établissements scolaires donnent 3'534 employés et les enquêteurs 2'628, mais 1'859 familles seulement se retrouvent simultanément dans les deux sources, soit $52 \%$ de celles classées comme telles par l'administration, et $70,7 \%$ de celles retenues par les enquêteurs.

La faible fiabilité des statistiques scolaires m'avait conduit (Prost, 1986), pour tenter de répondre à la question posée, à mener une enquête monographique, par coupes successives, de la Libération à 1980-81, dans tous les établissements de l'agglomération orléanaise, en saisissant en clair les professions des parents, pour les coder toutes ensemble et systématiquement de façon uniforme. D'autres enquêtes respectent les mêmes précautions méthodologiques, notamment celle de M. Duru-Bellat et A. Mingat $(1985,1988,1997,1999)$ sur une cohorte d'élèves entrés en 1982 en $6^{\text {ìme }}$ dans 17 collèges de l'académie de Dijon. Nous les utiliserons ainsi que les enquêtes "Formation, qualification professionnelle» (ou FQP) conduites périodiquement par l'INSEE (Institut National de la Statistique et des Études Économiques) et dont les données sur le niveau de formation et la position sociale sont relativement fiables.

On passera plus vite ici sur une troisième difficulté, plus technique, qui est liée aux outils statistiques utilisés pour comparer des écarts mesurés par des pourcentages et dire s'ils se réduisent ou s'aggravent. Faire leur différence arithmétique est commode, mais trompeur. Supposons deux groupes sociaux qui améliorent tous deux leur poids dans un niveau scolaire d'un même nombre de points, $8 \%$ par exemple, mais que l'un passe de 80 à $88 \%$, et l'autre de 4 à $12 \%$; il serait difficile de conclure qu'ils ont tous les deux également progressé: le second a triplé son poids relatif, quand le premier l'a augmenté de $10 \%$ seulement. Mais cette seconde appréciation, qui repose sur le rapport entre le taux final et le taux initial, n'est pas satisfaisante, car le premier groupe ne pouvait évidemment pas tripler son poids relatif, puisqu'il était déjà à $80 \%$ et ne pouvait, au mieux, 
progresser que d'un quart. Pour surmonter ces inconvénients, il faudrait raisonner non sur les poids relatifs des divers groupes sociaux dans les filières du système éducatif, mais sur la proportion d'enfants des divers groupes sociaux qui accèdent à ces filières, ce qui permettrait en outre de prendre en compte l'évolution considérable du poids relatif des différents groupes dans une société où les ouvriers diminuent, tandis que les cadres augmentent sensiblement. De telles chances d'accès ont été calculées par Duru-Bellat et Kieffer (1999a) à partir des enquêtes FQP, mais les données ne couvrent malheureusement pas les vingt dernières années du siècle. Notre appréciation sera donc plus précise pour les années 1945-1980 que pour les années suivantes.

\section{La démocratisation de 1945 à nos jours}

Pour cette première période, nous disposons d'abord de deux séries sur le poids des divers groupes sociaux à certains niveaux du système éducatif: une série nationale, extraite des publications du ministère, et la série orléanaise que nous avons construite. La seconde, qui repose sur une nomenclature homogène, nous paraît plus fiable que la première. Les tableaux 2 et 3 ci-dessous résument les résultats enregistrés par les deux groupes sociaux extrêmes, les cadres supérieurs et professions libérales d'une part, et les ouvriers de l'autre. ${ }^{10}$

Tableau 2: Part (en \%) des élèves des groupes sociaux extrêmes au niveau de la $2^{\text {de }}$ (série nationale, enseignement public)

\begin{tabular}{|lrrrrrr|}
\hline & $1961-62$ & $1963-64$ & $1967-68$ & $1973-74$ & $1976-77$ & $1980-81$ \\
\hline Ensemble des classes de 2de & & & & & & \\
$\quad$ Cadres supérieurs & 14,5 & 12,7 & 15,5 & 15,4 & 16,8 & 17,7 \\
$\quad$ Ouvriers & 20,3 & 23,0 & 27,1 & 27,8 & 28,6 & 30,2 \\
$\begin{array}{l}\text { de d'enseignement général } \\
\quad \text { Cadres supérieurs }\end{array}$ & 17,6 & 16,4 & 20,3 & 21,5 & 24,6 & 25,4 \\
$\quad$ & 14,9 & 17,3 & 22,1 & 21,8 & 20,7 & 23,0 \\
$\quad \begin{array}{l}\text { Ouvriers } \\
2^{\text {de }} \text { d'enseignement technique }\end{array}$ & & & & & & \\
$\quad$ Cadres supérieurs & 5,7 & 5,2 & 8,1 & 8,3 & 9,3 & 10,6 \\
$\quad$ Ouvriers & 35,5 & 34,6 & 34,6 & 34,6 & 35,5 & 36,6 \\
\hline
\end{tabular}

La première série suggère que les deux groupes sociaux extrêmes ont tous deux amélioré leur part relative dans la première classe des lycées, celle de $2^{\mathrm{de}}$, mais les ouvriers plus que les cadres supérieurs ${ }^{11}$, et dans les classes qui conduisent au baccalauréat général plus que dans celles du baccalauréat de technicien. Un regard plus attentif montre un léger recul des cadres entre 1961/62 et 1963/64, mais il est trop faible pour qu'on puisse en tirer argument, compte tenu de l'imprécision des données. Au demeurant, commencée en 1961 pour évaluer la politique alors engagée, cette série ne permet pas d'en comparer les effets à ceux de la ségrégation entre lycées et CC que cette politique prétendait corriger. 
Tableau 3: Part (en \%) des élèves des groupes sociaux extrêmes en seconde et terminale (enquête orléanaise, enseignements publics et privés)

\begin{tabular}{|c|c|c|c|c|c|c|c|c|}
\hline & $\begin{array}{l}1947 \\
1949\end{array}$ & $\begin{array}{l}1952 \\
1954 \\
\end{array}$ & $\begin{array}{l}1957 \\
1959 \\
\end{array}$ & $\begin{array}{l}1962 \\
1964\end{array}$ & $\begin{array}{l}1967 \\
1968\end{array}$ & $\begin{array}{l}1973 \\
1974 \\
\end{array}$ & $\begin{array}{l}1976 \\
1977\end{array}$ & $\begin{array}{l}1980 \\
1981\end{array}$ \\
\hline \multicolumn{9}{|c|}{ Ensemble des classes de $2^{\text {de }}$} \\
\hline Cadres supérieurs & 22,0 & 11,2 & 12,6 & 13,0 & 14,7 & 19,5 & 19,2 & 20,8 \\
\hline Ouvriers & 15,6 & 24,6 & 25,3 & 24,2 & 22,8 & 23,3 & 24,5 & 24,2 \\
\hline \multicolumn{9}{|l|}{$2^{\mathrm{de}} \mathrm{d}$ 'enseignement général } \\
\hline Cadres supérieurs & 26,4 & 17,5 & 17,6 & 16,2 & 19,7 & 28,9 & 27,0 & 27,9 \\
\hline Ouvriers & 8,7 & 15,5 & 17,8 & 21,5 & 18,0 & 17,4 & 19,8 & 20,2 \\
\hline \multicolumn{9}{|c|}{$2^{\text {de }}$ d'enseignement technique } \\
\hline Cadres supérieurs & 3,2 & 3,4 & 3,5 & 5,1 & 7,8 & 9,9 & 11,0 & 11,8 \\
\hline Ouvriers & 45,2 & 35,6 & 38,9 & 31,1 & 29,5 & 29,3 & 29,5 & 29,4 \\
\hline \multicolumn{9}{|c|}{$\begin{array}{l}\text { Ensemble des classes terminales } \\
\text { d'enseignement général }\end{array}$} \\
\hline Cadres supérieurs & 27,7 & 28,0 & 20,4 & 18,8 & 18,3 & 22,5 & 28,8 & 30,0 \\
\hline Ouvriers & 7,4 & 10,1 & 14,5 & 16,3 & 18,6 & 18,6 & 15,5 & 18,3 \\
\hline
\end{tabular}

La seconde série, construite dans une perspective historique plus large, ne présente pas cet inconvénient. Elle a d'autre part l'avantage de porter à la fois sur les classes de $2^{\text {de }}$ et sur les classes terminales que la série nationale ignorait. Ses enseignements surprennent. Le poids relatif des ouvriers s'est en effet sensiblement amélioré avant la mise en œuvre de la politique de démocratisation de l'enseignement, et il s'est affaibli ou stabilisé après la réforme, qui n'a donc pas eu les effets qu'elle recherchait. En $2^{\text {de }}$ d'enseignement général, la proportion d'enfants d'ouvriers augmente jusqu'aux réformes de 1962/6412; elle dépasse alors celle des enfants de cadres supérieurs en recul de 10 points depuis 1947: la démocratisation semble nettement progresser. Par la suite, le poids des enfants d'ouvriers recule avant de s'améliorer en fin de période, sans retrouver un niveau aussi élevé, tandis que celle d'enfants de cadres supérieurs remonte et s'établit en 1980/81 à des niveaux supérieurs à ses niveaux initiaux. Les conclusions sont identiques pour les classes terminales, à la différence près, fort logique, d'un décalage de quelques années vers l'aval, le point de retournement se situant en 1967/68. ${ }^{13}$

Ces résultats n'emportent pourtant pas totalement la conviction. Ils ne tiennent pas compte, en effet, de l'évolution respective des groupes sociaux en question. Les confrontations effectuées avec l'évolution du poids de ces groupes dans la classe d'âge des parents ne les remettent pas en cause, mais il serait plus rigoureux de raisonner sur la proportion des enfants d'ouvriers ou de cadres qui parviennent en $2^{\text {de }}$ ou en terminale, c'est-à-dire sur des taux d'accès. C'est ce qu'ont fait notamment Duru-Bellat et Kieffer à partir de qui nous avons construit le tableau 4 ci-après (1999a, pp. 86-87). 
Tableau 4: Taux d'accès aux différents paliers des enfants de cadres et d'ouvriers

\begin{tabular}{|c|c|c|c|c|c|c|c|}
\hline \multirow[b]{2}{*}{ Entrée en $6^{\text {¿me }}$} & \multicolumn{7}{|c|}{ Dates auxquelles les élèves ont eu 11 ans: } \\
\hline & $\begin{array}{c}\text { Avant } \\
1940\end{array}$ & $\begin{array}{l}1940 \\
1949\end{array}$ & $\begin{array}{l}1950 \\
1959\end{array}$ & $\begin{array}{l}1960 \\
1964\end{array}$ & $\begin{array}{l}1965 \\
1969\end{array}$ & $\begin{array}{l}1970 \\
1974\end{array}$ & $\begin{array}{l}1975 \\
1984\end{array}$ \\
\hline $\begin{array}{l}\text { Ensemble de la génération } \\
\text { Enfants de cadres } \\
\text { Enfants d'ouvriers } \\
\text { Coefficient d'inégalité }\end{array}$ & $\begin{array}{l}26,7 \\
83,9 \\
19,8 \\
21,1 \\
\end{array}$ & $\begin{array}{l}24,1 \\
83,0 \\
16,2 \\
25,3 \\
\end{array}$ & $\begin{array}{l}36,9 \\
86,5 \\
23,7 \\
20,6 \\
\end{array}$ & $\begin{array}{l}46,7 \\
89,2 \\
33,9 \\
16,1 \\
\end{array}$ & $\begin{array}{l}75,4 \\
96,5 \\
67,8 \\
13,1 \\
\end{array}$ & $\begin{array}{c}91,6 \\
97,3 \\
87,5 \\
5,1 \\
\end{array}$ & $\begin{array}{r}96,1 \\
99,0 \\
93,2 \\
7,2 \\
\end{array}$ \\
\hline \multirow[b]{2}{*}{ Entrée en $2^{\text {de }}$} & \multicolumn{7}{|c|}{ Dates auxquelles les élèves ont eu 16 ans: } \\
\hline & $\begin{array}{c}\text { Avant } \\
1945\end{array}$ & $\begin{array}{l}1945 \\
1954\end{array}$ & $\begin{array}{l}1955 \\
1964\end{array}$ & $\begin{array}{l}1965 \\
1969\end{array}$ & $\begin{array}{l}1970 \\
1974\end{array}$ & $\begin{array}{l}1975 \\
1979\end{array}$ & $\begin{array}{l}1980 \\
1989\end{array}$ \\
\hline $\begin{array}{l}\text { Ensemble de la génération } \\
\text { Enfants de cadres } \\
\text { Enfants d'ouvriers } \\
\text { Coefficient d'inégalité }\end{array}$ & $\begin{array}{r}12,1 \\
64,8 \\
5,4 \\
32,4\end{array}$ & $\begin{array}{r}13,1 \\
69,4 \\
5,9 \\
36,2\end{array}$ & $\begin{array}{l}24,3 \\
81,6 \\
11,4 \\
34,5\end{array}$ & $\begin{array}{l}28,2 \\
80,9 \\
14,9 \\
24,2\end{array}$ & $\begin{array}{l}34,5 \\
81,5 \\
19,0 \\
18,8\end{array}$ & $\begin{array}{l}40,4 \\
84,7 \\
23,2 \\
18,3\end{array}$ & $\begin{array}{l}49,2 \\
87,4 \\
28,0 \\
17,8\end{array}$ \\
\hline $\begin{array}{l}\text { Pour } 100 \text { enfants entrés en } 6^{\text {eme }} \\
\text { Enfants de cadres } \\
\text { Enfants d'ouvriers } \\
\text { Coefficient d'inégalité }\end{array}$ & $\begin{array}{r}45,3 \\
77,2 \\
27,0 \\
9,2 \\
\end{array}$ & $\begin{array}{r}54,5 \\
83,6 \\
36,5 \\
8,9 \\
\end{array}$ & $\begin{array}{l}65,8 \\
90,8 \\
47,8 \\
10,8 \\
\end{array}$ & $\begin{array}{r}60,5 \\
88,6 \\
48,1 \\
8,1 \\
\end{array}$ & $\begin{array}{l}45,7 \\
84,4 \\
28,0 \\
13,9 \\
\end{array}$ & $\begin{array}{l}42,7 \\
84,7 \\
25,9 \\
15,8\end{array}$ & $\begin{array}{l}51,2 \\
88,2 \\
30,1 \\
17,4 \\
\end{array}$ \\
\hline \multirow[b]{2}{*}{ Obtention du bac général } & \multicolumn{7}{|c|}{ Dates auxquelles les élèves ont eu 19 ans: } \\
\hline & $\begin{array}{c}\text { Avant } \\
1948\end{array}$ & $\begin{array}{l}1948 \\
1957\end{array}$ & $\begin{array}{l}1958 \\
1967\end{array}$ & $\begin{array}{l}1968 \\
1972 \\
\end{array}$ & $\begin{array}{l}1973 \\
1977\end{array}$ & $\begin{array}{l}1978 \\
1982 \\
\end{array}$ & $\begin{array}{l}1983 \\
1992\end{array}$ \\
\hline $\begin{array}{l}\text { Ensemble de la génération } \\
\text { Enfants de cadres } \\
\text { Enfants d'ouvriers } \\
\text { Coefficient d'inégalité }\end{array}$ & $\begin{array}{c}5,0 \\
35,1 \\
1,2 \\
44,5\end{array}$ & $\begin{array}{c}5,7 \\
39,9 \\
1,5 \\
43,6\end{array}$ & $\begin{array}{c}12,2 \\
50,3 \\
4,0 \\
29,5\end{array}$ & $\begin{array}{l}12,7 \\
56,2 \\
5,0 \\
24,5\end{array}$ & $\begin{array}{c}16,7 \\
59,7 \\
6,0 \\
23,2\end{array}$ & $\begin{array}{c}21,6 \\
62,7 \\
8,7 \\
17,6\end{array}$ & $\begin{array}{l}28,3 \\
65,5 \\
11,9 \\
14,1\end{array}$ \\
\hline $\begin{array}{l}\text { Pour } 100 \text { enfants entrés en } 6^{\text {ème }} \\
\text { Enfants de cadres } \\
\text { Enfants d'ouvriers } \\
\text { Coefficient d'inégalité }\end{array}$ & $\begin{array}{c}18,8 \\
41,8 \\
6,0 \\
11,3\end{array}$ & $\begin{array}{c}23,6 \\
48,0 \\
10,5 \\
7,9\end{array}$ & $\begin{array}{c}33,1 \\
58,2 \\
19,5 \\
5,7\end{array}$ & $\begin{array}{c}27,2 \\
53,8 \\
17,1 \\
5,6\end{array}$ & $\begin{array}{c}22,1 \\
61,9 \\
9,0 \\
16,4\end{array}$ & $\begin{array}{c}22,9 \\
62,7 \\
9,5 \\
16,0\end{array}$ & $\begin{array}{l}29,5 \\
66,1 \\
12,8 \\
13,3\end{array}$ \\
\hline
\end{tabular}

Selon cette source, les progrès de la démocratisation semblent globalement continus. Dans le tableau 4, les lignes intitulées: "coefficient d'inégalité» sont particulièrement intéressantes, car elles expriment, par un coefficient simple, si l'écart entre les cadres et les ouvriers se réduit ou s'accroît. ${ }^{14}$ Un coefficient de 1 traduirait une égalité complète des chances. La démocratisation quantitative, provoquée par la généralisation de la scolarité en collège se reflète clairement dans ces chiffres, avec, en $6^{\text {ème }}$, des taux d'accès pour les enfants d'ouvriers en fin de période supérieurs à $93 \%$, et un coefficient d'inégalité qui passe de 21,1 à 5 ou 7. A l'entrée du lycée, en classe de $2^{\mathrm{de}}$, les écarts se sont également réduits, même s'ils restent forts, avec des taux d'accès qui ont progressé de $5,4 \%$ à $28 \%$ pour les enfants d'ouvriers, tandis que ceux des enfants de cadres passaient de $64,8 \%$ à $87,4 \%$. Le coefficient d'inégalité diminue de 32,4 à 17,8 , ce qui indique une égalisation relative, même si les différences restent très fortes. On relève la même évolution pour l'accès au baccalauréat d'enseignement général. La démocratisation semble donc bien en marche. 
Ces résultats semblent contredire l'enquête orléanaise, qui montrait deux mouvements inverses, une réduction des inégalités de la Libération au milieu des années ' 60 , et un recul, ou du moins une stagnation de la démocratisation au cours des quinze années suivantes. En fait, apparemment contradictoires, les deux conclusions sont complémentaires. La démocratisation constatée est due essentiellement à la généralisation de l'accès en $\sigma^{\text {ème: }}$ or l'enquête orléanaise ne concerne, par définition, que des élèves qui ont franchi ce seuil et elle porte, non pas sur l'accès global des enfants d'ouvriers à des scolarités longues, qui s'est effectivement amélioré, mais sur leur poids relatifs dans les différentes filières de cette scolarité longue. Il est tout à fait possible que les écarts sociaux entre filières se soient accrus, alors que le mouvement général était à la démocratisation. Ce qui conduit Merle (2000) à proposer les concepts paradoxaux de démocratisation ségrégative, uniforme ou égalisatrice, pour désigner, à l'intérieur d'une démocratisation quantitative globale, l'accroissement des écarts sociaux entre filières, leur maintien ou leur réduction.

On voit alors tout l'intérêt de se placer aux points de bifurcation du système scolaire pour voir si les inégalités s'accroissent ou se réduisent entre les élèves qui ont franchi ces paliers (Duru-Bellat, Jarousse \& Mingat, 1993; Duru-Bellat \& Kieffer, 1999a). Or l'examen, dans le tableau 4, de l'évolution des chances d'accès en seconde pour les enfants entrés en $6^{\text {ime }}$, montre une légère réduction des inégalités jusqu'au milieu des années soixante, un peu accusée pour les élèves qui ont bénéficié de l'ouverture massive des $\sigma^{\text {eme }}$ entre 1960 et 1964, suivie d'une détérioration particulièrement sensible pour les élèves qui ont eu 16 ans entre 1970 et 1974 , c'est-à-dire au moment précis où se met en place la procédure contraignante d'orientation qui a pour effet immédiat de creuser les écarts entre groupes sociaux, et donc d'entraîner ce que Merle appelle une démocratisation ségrégative. Au niveau du baccalauréat d'enseignement général, il en va de même, à l'exception de la dernière période: les inégalités, qui se creusent fortement de 196872 (avant la mise en place d'une orientation contraignante) à 1978-82, reculent pour les élèves qui atteignent le baccalauréat entre 1982 et 1993. Les changements morphologiques provoqués dans l'enseignement professionnel par la réforme de 1985 ont en effet probablement rendu plus faciles les passages des enseignements techniques et professionnels vers l'enseignement général, ce qui expliquerait une démocratisation plus égalisatrice qu'au cours de la période précédente.

L'évolution depuis 1985 reste très difficile à apprécier. La croissance massive des lycées a probablement provoqué une démocratisation de l'accès en seconde, tandis que l'ouverture de la filière conduisant au baccalauréat professionnel a sans doute permis à des enfants d'ouvriers de parvenir en plus grand nombre au baccalauréat. Mais, pour mesurer cette réduction probable des inégalités, les indicateurs sont fragiles. Les enquêtes FQP n'ont pas assez de recul. Restent les études longitudinales du ministère sur des panels d'élèves entrés en $\sigma^{\text {ime }}$ à diverses dates. Pour l'accès au baccalauréat, la comparaison entre le panel 1972 et le pa- 
nel 1980 suggère une légère démocratisation, le coefficient d'inégalité passant de 9,7 à 8,4 entre les deux dates (Euriat \& Thélot, 1995). Pour l'accès en classe de $2^{\mathrm{de}}$, la comparaison peut porter également sur le panel 1989 et elle suggère une plus forte démocratisation dans la dernière période, avec des coefficients d'inégalité qui passent, pour les trois panels, de 16,9 à 16,2 puis à 12,4 (Duru-Bellat \& Kieffer, 1999a, p. 183). On aurait ainsi trois grandes phases historiques: de la Libération au milieu des années soixante, une démocratisation égalisatrice, puis, jusqu'en 1985, une démocratisation ségrégative, suivie de nouveaux progrès et probablement une démocratisation uniforme.

Mais la conclusion globale demeure plutôt négative en ce qui concerne les politiques scolaires. Au terme d'une étude sophistiquée, où ils croisent la position sociale obtenue, le diplôme et l'origine sociale, Goux et Maurin (1997) concluent que l'expansion scolaire contemporaine ne s'accompagne pas d'une réduction notable de l'inégalité des chances. C'est un constat qui s'est imposé très tôt aux acteurs du système éducatif, et qui a entraîné le déplacement des thématiques de la démocratisation, voire leur abandon.

Dès le milieu des années soixante-dix, en effet, l'espoir de transformer la société par une réforme des structures de l'enseignement s'estompe devant les réalités. Boudon (1973) argumente l'impossibilité de faire correspondre la structure scolaire et la structure sociale. Il montre comment, à chaque bifurcation du système scolaire, le poids du milieu social intervient de façon décisive, à la fois par le niveau de réussite des élèves et par le choix des familles. Les enseignants, comme les analystes, se heurtent à la résistance des inégalités et constatent que la réussite scolaire elle-même dépend de l'origine sociale: dès l'école élémentaire, la proportion de bons élèves n'est pas aussi forte parmi les enfants d'ouvriers que de cadres. Il ne suffit donc pas de lutter contre les inégalités d'orientation à réussite scolaire égale, ce qui était l'enjeu de la démocratisation des années ‘60, quand elle était une démocratisation de la sélection; il faut aussi, et sans doute d'abord, lutter contre les inégalités de réussite. Apparaît alors le thème du handicap socioculturel, qui invoque les travaux de linguistes comme Bernstein (trad. 1975), et celui des pédagogies de soutien ou de compensation. La généralisation de la préscolarisation trouve ici une justification sociale parce qu'elle améliore la réussite à l'école élémentaire des élèves défavorisés. Le thème de la lutte contre l'échec scolaire et de l'école de la réussite domine les années ' 80 , l'ambition de l'égalité des résultats remplace celle de l'égalité des chances: face à un objectif aussi exigeant, les progrès ne peuvent paraître que décevants. Le grand mouvement qui soulevait l'école d'après-guerre en faveur d'une réforme dont on attendait l'avènement d'une société plus juste et plus égalitaire est retombé. Le passage de la démocratisation à la lutte contre l'échec scolaire est ainsi l'histoire d'un désenchantement, qui annonce peut-être un recentrage modeste de l'école sur des objectifs purement scolaires. 


\section{Notes}

1 En 1919, dans l'article de Félix Pécaut, fils du fondateur de l'Ecole Normale Supérieure (ENS) de Fontenay-aux-Roses, "Ecole unique et démocratisation», Revue pédagogique, 74 (4), avril 1919, 235-252 (Briand \& Chapoulie, 1992, p. 408).

2 Rappelons qu'en France, les classes successives sont numérotées à partir de la fin de la scolarité. L'enseignement du second degré commence donc à la classe de $6^{\mathrm{ime}}$; la première partie du baccalauréat (jusqu'en 1965, où elle est supprimée), se passe à la fin de la classe de $1^{\text {ire }}$ et elle est suivie d'une classe dite «terminale».

3 Archives du lycée Marceau, cité par Prost, 1997, p. 91.

4 De 1873 à 1965, le baccalauréat était divisé en deux parties, la première se passant à la fin de la classe de $1^{\text {rec }}$ (ou rhétorique), et la seconde à la fin de la classe terminale (philosophie ou mathématiques élémentaires, ou encore, à partir de 1942, philosophie-sciences).

5 Respectivement collèges d'enseignement secondaire (CES) et collèges d'enseignement général (CEG).

6 Les deux filières modernes furent unifiées en 1969 et le report de l'initiation au latin en deuxième année du collège unifia la première année de la filière classique et de la filière moderne. D'autres mesures purement administratives permirent aux chefs d'établissements issus du primaire de diriger des collèges de type secondaire.

7 L'effectif de ces classes élémentaires décroît à partir de 1956-57.

8 Sondage IFOP confidentiel, demandé par le ministre et remis le 26 mai 1982. Archives Savary 3SV5.

9 L'évolution est trop forte en un laps de temps trop court, et elle correspond trop bien aux intérêts de l'administration à l'époque, pour que le soupçon ne s'impose pas.

10 Pour les précisions sur l'établissement de ces séries, on se reportera à Prost, 1986.

11 Par l'expression cadres supérieurs, on entendra toujours ici l'ensemble du groupe cadres supérieurs et professions libérales.

12 La population de l'enquête est celle des deux années scolaires successives, pour améliorer la fiabilité des résultats.

13 L'analyse reposant sur une succession de coupes transversales, elle ne permet pas de dater plus précisément les années où la tendance se retourne.

14 Ces chiffres, nommés «chances relatives» par Duru-Bellat et Kieffer, sont des odds ratio . Il s'agit d'un rapport de rapports: le rapport entre les probabilités d'accès vs/les probabilités contraires pour les cadres sur le même rapport pour les ouvriers. Le fait d'intégrer dans le calcul les probabilités de non-accès permet de réduire les biais induits par la proximité ou l'éloignement des $100 \%$.

\section{Références bibliographiques}

Bernstein, B. (1975). Langage et classes sociales. Paris: Ed. de Minuit.

Boudon, R. (1973). L’inégalité des chances, la mobilité sociale dans les sociétés industrielles. Paris: A. Colin.

Bourdieu, P. \& Passeron, J.-C. (1964). Les héritiers. Les étudiants et la culture. Paris: Ed. de Minuit.

Briand, J.-P. \& Chapoulie, J.-M. (1981). L'enseignement primaire supérieur des garçons en France, 1918-1942. Actes de la recherche en sciences sociales, 39, 87-111.

Briand, J.-P. \& Chapoulie, J.-M. (1992). Les collèges du peuple. Paris: CNRS/INRP/ENS de Fontenay, St. Cloud.

Duru-Bellat, M., Jarousse, J. P. \& Mingat, A. (1993). Les scolarités de la maternelle au lycée. Etapes et processus dans la production des inégalités sociales. Revue française de sociologie, XXXIV, (1), 43-60. 
Duru-Bellat, M. \& Kieffer, A. (1999a). La démocratisation de l'enseignement «revisitée». Dijon: Irédu (=Les Cahiers de l'irédu, ${ }^{\circ} 60$ ).

Duru-Bellat, M. \& Kieffer, A. (1999b). Evaluer la démocratisation de l'enseignement: la situation française à l'épreuve des comparaisons internationales. Revue française de pédagogie, 127, 49-61.

Duru-Bellat, M. \& Mingat, A. (1985). De l'orientation en fin de $5^{\mathrm{eme}}$ au fonctionnement $d u$ collège. Evaluation de la procédure. Dijon: Irédu (=Les Cahiers de l'irédu, $\left.\mathrm{n}^{\circ} 42\right)$.

Duru-Bellat, M. \& Mingat, A. (1988). De l'orientation en fin de $5^{\text {eme }}$ au fonctionnement $d u$ collège. Progression, notation, orientation: l'influence du contexte de scolarisation. Dijon: Irédu (=Les Cahiers de l'irédu, $\mathrm{n}^{\circ} 45$ ).

Duru-Bellat, M. \& Mingat, A. (1997). La constitution de classes de niveau dans les collèges: les effets pervers d'une pratique à visée égalisatrice. Revue française de sociologie, XXXVIII, (4), 751-759.

Duru-Bellat, M. \& Mingat, A. (1999). How Do French Junior Secondary Schools Operate? Academic Achievement, Grading and Streaming of Students. In A. Leschinsky \& K. U. Mayer (Ed.), The Comprehensive School Experiment Revisited: Evidence from Western Europe (pp. 64-92). Frankfurt/M.: Peter Lang.

Euriat, M. \& Thélot, C. (1995). Le recrutement social de l'élite scolaire depuis quarante ans. Education \& formations, 41, 3-20.

Gavoille, J. (1983). L'obligation scolaire un quart de siècle après Jules Ferry: le législateur face aux réalités. In W. Frijhoff (Ed.), L'offre d'école (pp. 341-355). Paris: Publications de la Sorbonne \& INRP.

Girard, A. \& Bastide, H. (1963). La stratification sociale et la démocratisation de l'enseignement, Population, 1963, (3), 435-472.

Goux, D. \& Maurin, E. (1997). Démocratisation de l'école et persistance des inégalités. Economie et statistique, 306, 27-39.

Langouet, G. \& Leger, A. (1991). Public ou privé? Trajectoires et réussites scolaire. Paris: Publidix.

Merle, P. (2000). Le concept de démocratisation de l'institution scolaire: une typologie et sa mise à l'épreuve. Population, 55, (1), 15-50.

Merllié, D. (2000). La confrontation de deux sources d'information sur la catégorie socio-professionnelle des parents d'élèves. Population, 55, (1), 181-188.

Poignant, R. (1965). L'enseignement dans les pays du marché commun. Paris: Institut pédagogique national.

Prost, A. (1981). L'école et la famille dans une société en mutation (1930-1980). Paris: Nouvelle librairie de France [Parias, L.-H. (Dir.). Histoire générale de l'enseignement et de l'éducation en France, tome IV].

Prost, A. (1986). L'enseignement s'est-il démocratisé? Paris: PUF.

Prost, A. (1992), L'enseignement s'est-il démocratisé? (2. Ed. rev. et augm.). Paris: PUF.

Prost, A. (1997). Education, société et politiques. Une histoire de l'enseignement de 1945 à nos jours. Paris: Seuil.

Prost, A. (1998). L'Education nationale depuis la Libération. Cahiers français, (285), 3-12.

Talbott, J. (1969). The Politics of Educational Reform in France. Princeton: Princeton University Press. 


\section{Die Demokratisierung des Unterrichts in Frankreich seit dem Zweiten Weltkrieg}

\section{Zusammenfassung}

Dieser Artikel bilanziert die Demokratisierung des Unterrichts in Frankreich. Er erinnert an die Ursprünge dieser Politik in der ersten Hälfte des 19. Jahrhunderts und zeigt, dass die Realisierung, die erst in den Jahren 1959-63 erfolgte, mit der Reform der Sekundarstufe I (l'école moyenne) und der ersten Welle des Wachstums zusammenhängt. Anschliessend analysiert der Beitrag die zweite Welle des Wachstums, welche die Gestalt des Gymnasiums nach 1985 fundamental veränderte. Es stellt sich die Frage nach den Resultaten dieser Prozesse: Hat die entsprechende Politik die sozialen Ungleichheiten gegenüber der Schule reduziert?

Die Antwort muss differenziert ausfallen: Die Öffnung der weiterführenden Schulen (collège und lycée) hat den Zugang für Kinder aus bescheidenen Verhältnissen erleichtert und allgemein die Ungleichheiten reduziert. Aber unterhalb dieses Gesamtzusammenhanges hat sich die Ungleichheit zwischen den einzelnen Schultypen auf unterschiedliche Art und Weise entwickelt: Bis Mitte der 1960er-Jahre ist die Ungleichheit geringer geworden. Der Demokratisierungsprozess ist anschliessend zum Stillstand gekommen, wenn nicht wegen der 1973 eingeführten selektiven Prozeduren sogar ausgehölt worden. Die Entscheide von 1985 haben dagegen den Demokratisierungsprozess wieder in Gang gebracht. Aber die Hoffnungen, die damit verbunden waren, sind insgesamt nicht erfüllt worden.

\section{La democratizzazione dell'insegnamento in Francia dopo la Seconda Guerra mondiale}

\section{Riassunto}

Questo articolo traccia un bilancio della democratizzazione dell'insegnamento in Francia. Ricorda le origini di questa politica durante la prima metà del secolo e spiega perché tale politica ha potuto essere realizzata negli anni 1959-63: è questo infatti il periodo della riforma della scuola media e della prima ondata di crescita fino a metà degli anni '70. Inoltre l'articolo analizza la seconda ondata di crescita che rivoluziona la morfologia del liceo a partire dal 1985. A questo punto pone la questione dei risultati: queste politiche hanno ridotto le disuguaglianze sociali rispetto alla scuola?

La risposta è sfumata. L'apertura della scuola media e poi dei licei ha facilitato l'accesso agli studi degli allievi di origine modesta e globalmente ha ridotto le disuguaglianze. Ma, all'interno di quest' evoluzione d'assieme, l'ineguaglianza tra le diverse "filières» si è sviluppata in maniere variabili: fino alla metà degli anni ' 60 sembra essersi ridotta, in seguito si è stabilizzata o addirittura è aumentata in ra- 
gione delle procedure di selezione e degli orientamenti introdotti nel 1973. Le decisioni del 1985 infine hanno senza dubbio rilanciato la democratizzazione. Eppure le speranze di cui si era fatta carico sono andate deluse.

\section{The democratization of instruction in France since the Second World War}

\section{Summary}

This article takes stock of the democratization of education in France. It reminds one of the origins of this policy in the first half of the nineteenth century and shows that the realization, that only followed in the years 1959-1963, is connected with the reform of secondary level I (école moyenne) and the first wave of growth. Following this, the paper analyzes the second wave of growth that fundamentally changed the shape of the gymnasium after the year of 1985. Finally, the question of the results of this process is raised: Did the respective policy reduce the social inequality towards school?

The answer must be variegated: The opening of continued schools (collège and lycée) has eased the access for children from modest circumstances and generally reduced inequalities. But below this commonality, the inequality between individual schools has developed in various ways: Until the mid-1960's, the inequality diminished. After this process of democratization, it came to a halt, if not even hollowed out by the selective procedures introduced in 1973. The decisions of 1985, however, put the process of democratization back in gear. But the connected hopes, overall, were not fulfilled. 